\title{
Switching clotting factor concentrates: considerations in estimating the risk of immunogenicity
}

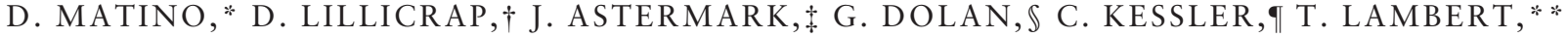

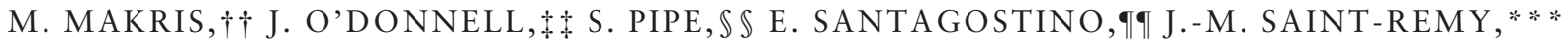 \\ W. SCHRAMM $+\dagger \dagger$ and A. IORIO ++ + \\ *Experimental Medicine and Biochemical Sciences, University of Perugia, Perugia, Italy; $\dagger$ Department of Pathology and \\ Molecular Medicine, Richardson Laboratory Queen's University, Kingston, ON, Canada; $\$$ Department of Hematology and \\ Coagulation Disorders, Skåne University Hospital Malmö/Lund, Malmö, Sweden; \$University Hospital Queen's Medical \\ Centre, Nottingham, UK; $\uparrow$ Georgetown University Medical Center Lombardi Cancer Center, Washington, DC, USA; **Centre \\ de Traitement des Hémophiles Hôpital de Bicêtre AP-HP, Le Kremlin Bicêtre, France; ††Department of Cardiovascular \\ Science, University of Sheffield Royal Hallamshire Hospital, Sheffield, UK; \$+National Centre for Hereditary Coagulation \\ Disorders St James's Hospital \& Trinity College Dublin, Dublin, Ireland; $\$ \$ \$$ Department of Pediatrics Division of Pediatric \\ Hematology/Oncology, University of Michigan, Ann Arbor, Michigan, USA; $\mathbf{q}$ Angelo Bianchi Bonomi Haemophilia and \\ Thrombosis Centre of the IRCCS Maggiore Hospital Mangiagalli and Regina Elena Foundation, Milan, Italy; ***Department \\ of Immunology, University of Leuven, Leuven, Belgium; ††Department of Transfusion Medicine and Haemostasis, University \\ Hospital of Munich, Munich, Germany; and \$+Department of Clinical Epidemiology and Biostatistics, McMaster University \\ Hamilton, Ontario, Canada
}

Summary. The development of neutralizing antibodies to factor VIII (FVIII) is the most serious complication of therapy for haemophilia A. There is now excellent documentation that a large number of both genetic and environmental factors contribute to the risk of FVIII inhibitor incidence. One of the environmental factors that has been proposed as an influence on this complication is the occurrence of FVIII product switching. There are only a small number of clinical studies that have addressed this question, and thus, the amount of objective information available to assess this association is limited. In this review, in addition to summarizing past evidence pertinent to this subject, we present the results of a complementary strategy, a Delphi analysis, to add to the considerations of product switching and FVIII immunogenicity. With the imminent arrival in the clinic of several new FVIII products, the haemophilia community must be prepared to collect prospectively controlled data to better address this important management issue.

Keywords: clotting factor concentrates, haemophilia, immunogenicity, inhibitors

\section{Introduction}

Current protein replacement therapies for haemophilia are well documented to be haemostatically effective and safe [1]. Since the catastrophic events of the mid1980s, the risk of infectious agent transmission has become essentially theoretical, thus leaving the inherent immunogenicity of the clotting factor concentrates as the principal treatment-related complication of hae-

Correspondence: David Lillicrap, Professor, Department of Pathology and Molecular Medicine, Richardson Laboratory, Queen's University, Kingston, Ontario, Canada, K7L 3N6. Tel.: +613 548 1304; fax: +613 548 1356; e-mail: dpl@queensu.ca

Accepted after revision 17 September 2013 mophilia therapy. This is a particular concern in haemophilia A [2].

The pathogenesis and biology of the factor VIII (FVIII) immune response has been a subject of extensive investigation for the past three decades, and some facts are now well established. The cumulative incidence of functionally neutralizing FVIII antibodies (FVIII inhibitors) in previously untreated patients (PUPs) is approximately $30 \%$ and these antibodies usually develop during the first 10-20 exposures to FVIII exposure [3]. In contrast, in patients who have been repeatedly treated with FVIII concentrates ( $>150$ exposures-previously treated patients [PTPs]), the baseline inhibitor risk is approximately $2-3$ per 1000 patient-years [4-6].

It is now well recognized that the risk of inhibitor development in PUPs is determined by a complex 
interaction between multiple genetic and environmental factors [7]. Among the genetic risk factors, the FVIII mutant genotype, ethnicity, and variants of several immunoregulatory genes have all been characterized to play a role as determinants of the inhibitor risk. Similarly, in terms of environmental risk determinants, the presence of intercurrent inflammation, intensity of treatment, surgery, the age of first FVIII exposure, FVIII dose and FVIII product type have all been evaluated as potential risk modifiers and have been found to contribute variably to this outcome.

In light of the significantly higher incidence of inhibitor development in PUPs, most of the studies addressing risk factors for FVIII immunogenicity have been conducted in this population, and not in PTPs. Nevertheless, the incidence of inhibitors does not disappear over the lifetime of the person with haemophilia (PWH) and there is recent evidence that in elderly PTPs there is a slight resurgence of this risk [8]. An examination of data relating to the baseline risk of inhibitors in PTPs shows that recent studies have documented a threefold increase in incidence (1.5-5.3 per/1000 patient-years). The cause of this observation remains unknown, but may be due to an increased awareness of inhibitors in this population and an increased testing frequency.

One of the factors that may influence inhibitor development in PTPs is the introduction of new FVIII concentrates. The role of product switching as a risk factor for FVIII immunogenicity has attracted attention in several studies in the past, and now with the introduction of several new FVIII products into the clinic over the next 5 years will again generate discussion.

\section{Immunological Considerations of FVIII Product Switching}

Assessing the risk of eliciting an immune response to FVIII when a new concentrate is introduced for a previously treated patient poses a number of interesting questions.

Patients with severe FVIII deficiency lack the capacity to eliminate high-affinity CD4+ T cells by induction of apoptosis in the thymus and, by the same token, lack the capability of generating natural Foxp3 $+\mathrm{T}$ regulatory cells to FVIII (although mechanisms of peripheral tolerance induction are intact). This scenario creates conditions under which any FVIII presenting even small structural alterations (to the primary amino acid sequence or to posttranslational modifications) is at risk of eliciting an immune response. These variances in structure can generate new B cell epitopes as well as new T-cell epitopes.

An important immunological attribute of FVIII is its potential for activating the innate immune system. Innate responses are not associated with immunologi- cal memory and thus, whatever the result of previous exposures to FVIII, each new administration is a new challenge to the innate immune system and carries a risk of eliciting an immune response. This may be all the more critical because the germinal configuration $\mathrm{B}$ cell repertoire contains cells that are capable of recognizing FVIII.

Algorithms are available to predict whether either new B or T-cell epitopes have been generated, with a predictive value as high as $80 \%$, when several of those algorithms are combined. In addition, a number of in vitro assays can be performed to determine whether potential new epitopes are present and of clinical relevance. Nevertheless, these types of analysis have not achieved widespread clinical application.

\section{Prior Experiences of FVIII Immunogenicity with Product Switching}

The potential for enhanced FVIII immunogenicity following product switching has been recognized for some time, and while the diversity of concentrates has increased over time, and will further expand over the next 5 years, the initial evidence that this phenomenon could occur concerns two inhibitor "outbreaks" 20 years ago in Belgium and the Netherlands involving a switch of plasma-derived concentrates $[9,10]$. In these two studies, a significant excess of inhibitors was documented in two cohorts of PTPs (up to fivefold increase; 20 per 1000 patient-years) with the introduction of plasma-derived FVIII (pd-FVIII) products that had undergone novel viral inactivation processes. It is assumed that these inactivation processes (long-duration pasteurization) resulted in structural changes to FVIII that initiated the subsequent immune responses. In contrast, all subsequent studies of FVIII product switching have not shown any evidence of enhanced immunogenicity (see Table 1).

Two Canadian studies have evaluated switches from pd-FVIII to recombinant FVIII (rFVIII) [11], and a later switch from first to second generation rFVIII in a total of $>700$ patients for up to 2 years [12]. Neither of these studies documented an increase in inhibitor incidence, and where new inhibitors were found they were always transient. In addition, these studies found evidence of pre-switch low titre inhibitors that had not been diagnosed at the treating clinic sites, highlighting one of the complicating issues in the interpretation of inhibitor test results around the time of product switching.

In the UK, a small study of 26 PTPs switching from intermediate to high purity pd-FVIII showed no increase in inhibitor risk [13] and another study of 33 PTPs switching from full-length FVIII to recombinant B-domain deleted FVIII showed just a single low titre inhibitor [14]. 
Table 1. Summary of clinical studies addressing the incidence of FVIII inhibitors associated with FVIII product switching.

\begin{tabular}{lll}
\hline References & $\begin{array}{l}\text { Patient number and } \\
\text { follow-up (months) }\end{array}$ & $\begin{array}{l}\text { Inhibitor rate } \\
\text { (per 1000 } \\
\text { patient-years) }\end{array}$ \\
\hline Giles et al. [11] & 478 (12 months) & 0.019 \\
& 339 (24 months) & 0.030 \\
Baglin et al. [13] & 26 & 0 \\
Singleton et al. [15] & 94 (<20 months) & 0.042 \\
Gouw et al. [17] & 104 (>50 exposure days) & 0 \\
Rubinger et al. [12] & 225 (12 months) & 0 \\
& 189 (24 months) & 0 \\
Rea et al. [14] & 33 (>3 months) & 0.033 \\
Siegmund et al. [23] & 118 & 0 \\
Bacon et al. [16] & 113 (>100 exposure days) & 0.009 \\
Gouw et al. [18] & 20 (pd-FVIII to rFVIII) & Adjusted HR 1.05 \\
& 94 (multiple FVIII brands) & (CI 0.44-2.49) \\
& $\quad$ Adjusted HR 0.99 & (CI 0.63-1.56) \\
\hline
\end{tabular}

CI, confidence interval; FVIII, factor VIII; HR, hazard ratio; pd-FVIII, plasma-derived factor VIII; rFVIII, recombinant factor VIII.

Two Irish studies, one evaluating inhibitors at the time of a switch from Chinese hamster ovary (ReFacto ${ }^{\circledR}$ ) to baby hamster kidney cell-derived (Kogenate ${ }^{\circledR}$ ) rFVIII (94 patients) [15] and the second, assessing the switch to a third generation full-length rFVIII (Advate $^{\circledR}$ ) in 113 patients both failed to show an excess of new inhibitors [16].

In the retrospective collection of inhibitor data in the CANAL study, 104 patients switched their FVIII product after a median of five exposures (range 2-48 exposures) [17]. The follow-up of this group showed a relative risk for inhibitor development of 0.9 (CI 0.6-1.6).

Most recently, as part of the RODIN study, 20 patients switched from a pd-FVIII product to rFVIII after a median of four exposure days and again the adjusted hazard ratio (HR) of 1.05 (confidence interval [CI] 0.44-2.49) indicated no evidence of increased inhibitor risk [18]. In this same study, 94 patients used multiple FVIII product brands. Seventy-one patients switched products once, 13 twice and 10 patients more often. Switching between different FVIII brands showed no enhanced risk of inhibitors (HR 0.99, CI 0.63-1.56).

Studies are ongoing in the UK and Australia following large scale switches from full-length rFVIII to a third generation B-domain deleted product. Preliminary results from these studies have shown no increased incidence of inhibitors following switching.

\section{Strategies for Evaluating the Risk of FVIII Immunogenicity Associated with Product Switching}

The currently available studies addressing this subject represent a mixture of methodological approaches. Several of the studies are retrospective in nature and thus prone to recall bias, and some of the studies are missing appropriate control groups. Overall, the amount and quality of data relating to the issue of
FVIII immunogenicity associated with product switching is modest.

In the text below, we have approached this question with an alternative methodology, using a modified Delphi technique. This process can be summarized as a structured group communication in which a complex problem (in this case, the risk of FVIII immunogenicity associated with product switching) can benefit from the subjective judgements of a number of experts. The process often, as in this case, begins with a face-to-face meeting to set the context of the communication and then proceeds through several rounds of questions and independent responses from the individual members of the expert group.

We believe that in the case of this subject matter, where the presence of high quality objective data is limited, that a Delphi approach adds useful additional information to the existing literature.

\section{Materials and methods}

A modified Delphi consensus process was used to reach the consensus statements presented in the following manuscript. An initial in person full day workshop was held in Toronto in September 2011. At this face-to-face meeting, each of the authors reviewed the available evidence on specific topics related to product switching and a series of presentations were given, followed by a general discussion. No recommendations were discussed during the meeting. After the meeting, two sequential Delphi rounds were performed. All of the authors, except Davide Matino, participated in the original face-to-face meeting and all rounds of the Delphi process. Mr. Matino was responsible for the analysis of the Delphi responses. The 12 expert panellists responded through an ad hoc web-based platform, and they were at all times blinded to individual votes from their colleagues. After each iteration of the Delphi survey, the panellists were presented with mean scores produced by the group. The first round of analysis aimed at identifying and ranking the items to be used as headings to organize the statements in the consensus paper. Ten items were initially proposed, asking the panellists to rank them in order of relevance. The 12 panellists were also asked to propose modifications of existing items, or to suggest the deletion of items or addition of new statements. After 3 iterations, an ordered list of 14 items was agreed upon, with consensus over $75 \%$ about both the wording and the ranking of the items. In the second round of the analysis, we proposed three statements for each of the 14 items and asked the panellists to score these statements. The panellists were asked to state for each statement their willingness to include or exclude the statement, and they were also prompted to propose modifications of existing statements or the addition of new ones. At each iteration, only items that were scored as "should 
include" by over $50 \%$ of panellists were proposed in subsequent rounds of the process. After 3 iterations, the scores became stable, and only the statements scored as "should include" by more than $60 \%$ of panellists have been kept in the final document.

Statements are reported for each item ordered by the descending level of consensus reached in the final iteration (consensus scores indicated in brackets).

\section{Results}

\section{Consensus items and statements}

Item 1. Evidence documenting an increased risk of FVIII inhibitor development with product switching is weak. A. The most direct evidence we have about the risk of inhibitor development after recombinant product switching consists of reports from Canada, UK and Ireland. From these studies, there is no clear signal of an increase in inhibitor development when switching to and from the currently available recombinant factor concentrates $(90 \%)$.

B. Some of the reluctance to change the type of concentrate that PWH are using has its origins in the past epidemics of blood-borne infections. In addition, inhibitor formation was observed following the introduction of two specific plasma-derived-FVIII concentrates in the 1990s. Little if any evidence points to an increased risk of inhibitor development with switching between different recombinant FVIII products. After products became safer, the frequency of real-world switching is much higher than actually appreciated $(66 \%)$.

Item 2. The risk of inhibitor development is likely to be less with FIX product switches compared to FVIII switches. A. The evidence about side effects of FIX treatment, with either plasma-derived or recombinant FIX, indicates an overall low risk of inhibitors. There is no evidence of an increased rate of inhibitors linked to switching $(100 \%)$.

B. Data about product switching in haemophilia B patients are far rarer than for haemophilia $\mathrm{A}$, due to the lower prevalence of the disease, lower incidence of inhibitors, and less availability of other concentrates to switch to and from $(83 \%)$.

C. The risk of inhibitors after switching from one brand of FIX to another is mostly unknown, but it is likely to be proportional to the overall risk of inhibitors to FIX, which is substantially lower than for FVIII $(75 \%)$.

Item 3. FVIII inhibitor development is more likely when product switches occur during the first 50 exposure days. A. In the absence of robust evidence about switching during the period of tolerization, it is considered beneficial, whenever possible, to keep patients on the same product for the first $50 \mathrm{ED}$. On the other hand, should the reason for switching be an important one, it has to be considered that no evidence exists against switching during this time $(92 \%)$.

B. We did not find any high quality evidence addressing this point. The CANAL study did not find any effect of switching on the rate of inhibitors in PUPs $(76 \%)$.

Item 4. The risk of FVIII inbibitor development may be increased with the new FVIII conjugates and fusion proteins. A. In theory, any change to the native FVIII protein could initiate an increase in immunogenicity. Careful observation for inhibitors will be required when introducing the new FVIII molecules (92\%)

B. No evidence is currently available in human beings about the immunogenic potential of these novel FVIII products. PEGylation, other forms of conjugation and fusion proteins have been used previously in humans without strong immune responses. All of these modifications could theoretically increase or reduce immunogenicity, and the new molecules have to be tested clinically to answer this question (75\%).

C. Bioengineering factor VIII could either enhance or reduce the immunogenicity of the protein $(69 \%)$.

Item 5. The risk of an inhibitor after switching may be different for severe vs. moderate or mild haemophilia. A. Mild or moderate haemophilia patients are usually not on prophylaxis, and in most cases the development of an inhibitor is associated with events triggering the use of high doses of factor (e.g. trauma or surgery); in these situations, the risk attributable to a product switch will be difficult to assess $(85 \%)$.

B. Mild and moderate haemophiliacs have a significantly lower risk of inhibitor development. Despite the known importance of the type of gene mutation in the inhibitor risk in this group of patients, the mechanisms by which they develop inhibitors is not fully characterized, so that surveillance is warranted after product switching $(77 \%)$.

C. Mild and moderate haemophiliacs have a significantly lower risk of inhibitor development, because they are tolerized by their own FVIII. The mechanisms by which this group of patients develop inhibitors are not well characterized, so that surveillance is warranted after product switching $(75 \%)$.

Item 6. The risk of FVIII inhibitor development with product switching is increased in patients with a past bistory of an inbibitor. A. The evidence for inhibitor development with product switching in patients with a past history of an inhibitor is weak and controversial. It is theoretically possible that changing the therapeutic molecule could break the tolerance to FVIII, but conversely we have anecdotal evidence of successful immune tolerance induction with a molecule different from that inducing the inhibitor $(77 \%)$. 
B. The evidence for inhibitor development with product switching in patients with a past history of an inhibitor is weak and controversial. It is theoretically possible that changing the therapeutic molecule could break the tolerance to FVIII, but conversely we have anecdotal evidence of successful immune tolerance induction with a molecule different from that inducing the inhibitor. We have also evidence of patients switched after success of immune tolerance, without relapse after switching $(69 \%)$.

C. Product registration studies usually exclude patients with a past personal history of inhibitors. A handful of cases of such patients have been switched in tender-related en masse switching exercises without any evidence of new high responding inhibitor formation $(67 \%)$.

D. There is no strong evidence for this statement. However, whenever a product switch is needed, strict surveillance is warranted $(61 \%)$.

Item 7. There may be an increased risk of inhibitor development when switching product just prior to surgery or intensive treatment. A. Even if not formally estimated in a rigorous way, the risk of inhibitors appears to be high after surgery and/or intensive treatment. There is no evidence relating to the contributory role of concomitantly switching FVIII (84\%).

B. Surgery and/or intensive FVIII treatment appears to be potent trigger to inhibitor formation. Whether a concomitant switch of product enhances the likelihood of inhibitor development in this setting remains unclear and will be difficult to evaluate $(84 \%)$.

Item 8. The risk of a FVIII inbibitor development increases with the frequency of product switching. A. The haemophilia population in countries where the supply of factor concentrates is determined by a tender system has undergone several switches. The measured increase in the inhibitor incidence, if any, lies in the order of $<3-5$ in $1000(84 \%)$.

B. There is no direct evidence to substantiate this statement, and information about the frequency of switching has never been systematically collected. The overall risk of inhibitor development after switching is very low and it is very unlikely that the risk can incorporate a clinically significant gradient associated with the number of previous switches $(77 \%)$.

Item 9. There is an increased risk of FVIII inhibitor development when switching concentrates in patients being treated on demand as opposed to prophylactically. A. The effect of treatment regimen on inhibitor development is controversial in PUPs and largely unknown in PTPs. There is no evidence to support or deny this statement $(85 \%)$.

B. There is no evidence to support this statement $(77 \%)$.
Item 10. There is an increased risk of FVIII inhibitor development when switching between plasma-derived and recombinant FVIII concentrates. A. There is no evidence to support this statement. The Canadian surveillance of product switching for the entire population to recombinant FVIII in 1988 did not show any increase in the baseline risk for inhibitor development $(92 \%)$.

B. There is no evidence that the risk of inhibitor development associated with switching from any plasma-derived concentrate to any recombinant product is different from that associated with switching between two different plasma-derived or two recombinant factor concentrates $(77 \%)$.

Item 11. There is an increased risk of FVIII inhibitor development when switching between B-domain deleted and full-length FVIII concentrates. A. The preliminary analysis of the prospective parallel observation of patients who switched or did not switch between these products in the UK population has not shown an increase in the inhibitor rate after switching to B-domain deleted FVIII (92\%).

B. The proper experiment to answer this question would be a crossover study, with PUPs randomized to one or the other molecule and then switched over when PTPs. Evidence from case series is likely to be biased and inconclusive $(83 \%)$.

C. There is no high level evidence to support this statement. A recent meta-analysis of observational trials suggests a difference, but several methodological and design flaws make the results of this study likely to be biased $(77 \%)$.

Item 12. FVIII inhibitor risk associated with recombinant FVIII use could be influenced by the type of cell employed for FVIII production. A. There is no evidence to support the statement. If any risk exists, this risk is low enough to be incorporated in the $<1$ per 1000 inhibitors observed in PTP populations (85\%).

B. The hypothesis is theoretically plausible; the type of cell used to produce recombinant FVIII influences the final structure of the molecule, for example, its degree and nature of glycosylation. We currently have no evidence to support or deny this statement $(77 \%)$.

Item 13. All recombinant FVIII products have the same risk of inhibitor development. A. Preliminary analysis of the EUHASS data shows a substantial equivalence of the risk of inhibitors in PUPs treated with any of the recombinant molecules. Most of the remaining evidence is made by uncontrolled case series not allowing any reliable comparison $(82 \%)$.

B. There is no evidence demonstrating a difference in immunogenicity between recombinant FVIII products (all registration trials show the same low inhibitor rate for all the currently available molecules). 
Robust evidence from prospective parallel observation does not yet exist $(77 \%)$.

C. The use of an antigenically modified plasmaderived molecule in 1993 in the Netherlands and Belgium was easily recognized to be associated with an increased risk for transient inhibitors. Until proven false, all the available recombinant molecules can be considered immunologically equivalent $(67 \%)$.

D. Nevertheless, differences in cell lines, carbohydrate modifications, and manufacturing processes cannot a priori be assumed to be equivalent in their immunogenicity before clinical trials are performed. The example of the increased immunogenicity of a structurally identical recombinant erythropoietin placed in a new vial design is an important reminder of this fact $(62 \%)$.

Item 14. The risk of FVIII inhibitor development with product switches can be predicted by genetic analysis. A. It is likely that mild haemophiliacs with dysfunctional FVIII molecules can be tolerant to their own FVIII but these patients may be prone to develop inhibitors to native (either recombinant or plasmaderived) FVIII protein administered for treatment. Genetic analysis may help identify this subgroup (92\%).

B. There is accumulating evidence that genetic analysis can predict the risk of inhibitor development in PUPs. How much this genetic evidence is relevant for predicting inhibitor risk in PTPs is largely unknown $(92 \%)$.

\section{Summary Statement and Future Studies}

The outcome of the Delphi process detailed above represents a complementary approach to providing information relating to the risk of FVIII inhibitor development associated with product switching. This information is not intended to supersede previous clinical data published on this subject, nor is it meant to diminish the value of ongoing prospective studies of this phenomenon. It is also important to recognize that the issue of FVIII immunogenicity is undergoing continual re-examination, and that while this Delphi process occurred at a specific point in time, there has been additional information published since completion of the Delphi review that merits consideration [18]. In this Delphi process, a mean consensus score of $80 \%$ was reached for the 36 statements reviewed.

There are currently a number of novel rFVIII concentrates that are undergoing late phase clinical trials, and several of these products will likely be licensed for more widespread clinical application in the next 2-3 years. All of these rFVIII proteins are modified in some aspect of their structure, as novel B-domain truncated molecules [19], as FVIII conjugates [20] or as fusion proteins [21]. There is evidence that all of these products provide some biological enhancement over native FVIII, most often some degree of prolongation of the FVIII half-life.

Clinicians treating PWH will need to consider the role of these new FVIII products for the management of their patient groups, and it is quite likely that decisions concerning product introduction will be highly variable based upon a variety of factors including geography, patient age, current product satisfaction and treatment regimen and cost. Potential product immunogenicity will also be a key consideration in the evaluation of these new treatments and prospective evaluation of this complication must be incorporated into future clinical practice.

As the haemophilia community moves forward, it is critical that more detailed information be gathered relating to FVIII product immunogenicity and the influence of product switching. Both switching and non-switching PWH should be prospectively enrolled in registries evaluating inhibitor incidence and inhibitor tests should be performed as a minimum, immediately prior to any product switch and for 2-3 months post switching. Examples of successful multinational registries documenting the incidence of adverse treatment outcomes are already in existence [22]. The organization of prospective, appropriately controlled surveillance programmes is a key element in the attainment of objectively useful information concerning this treatment complication.

\section{Acknowledgements}

This article is the outcome of an interactive face-to-face workshop meeting held in Toronto, Canada in 2011 and subsequent sequential rounds of Delphi panel and statistical analyses carried out at McMaster University, Ontario. Novo Nordisk Health Care AG, Switzerland sponsored the meeting, funded the research and provided administrative support. All authors, apart from David Matino, attended the face-to-face meeting and participated in all rounds of Delphi panel. DM and AI analysed the Delphi results. DL prepared the manuscript and all authors reviewed, revised and approved the final version.

\section{Disclosures}

D.M., J-M.S-R. and W.S. have no competing interests. D.L. has received research funding from Baxter Bioscience, Bayer and Biogen-Idec and has received honoraria for advisory board participation (Novo Nordisk, CSL Behring, Biogen Idec and Baxter). J.A. has received honoraria for advisory boards (Novo Nordisk, Pfizer, Baxter, CSL, Bayer) and lectures at meetings (Pfizer, SOBI, Bayer, Baxter, Novo Nordisk). He has received a research grant from Baxter. G.D. has received honoraria for speaking engagements and for attending advisory boards from Bayer, Baxter, Novo Nordisk, Pfizer, Biogen, Grifols and Biotest. C.M.K. has served as a paid consultant for Grifols, Baxter, Bayer, Pfizer, Novo Nordisk, Octapharma, CSL and Biogen. He has performed ed. research for Grifols, Baxter, Bayer, Novo Nordisk, Octapharma and Pfizer but no research funds benefit him directly and are received by his institution. T.L. has acted as a paid advisor to Baxter, Bayer, CSL, Novo Nordisk, Octapharma and Pfizer. M.M. has provided consultancy to Baxter, Bayer, Biogen, Biotest, BPL, Novo Nordisk, Octapharma, Pfizer and SOBI. He is the project leader for the EUHASS and EUHANET projects, which receives funding from Baxter, Bayer, Biotest, BPL, CSL Behring, Grifols, Kedrion, LFB, 
Novo Nordisk, Octapharma, SOBI/Biogen and Pfizer. J.S.O.D. has acted as a paid consultant to Octapharma, Baxter, Bayer and Pfizer. He has received funding for research from Baxter, Bayer and Novo Nordisk. S.W.P. received consultancy fees and travel reimbursement for attending a symposium. E.S. received fees as a speaker in meetings organized by Bayer, Baxter, Pfizer, CSL Behring, Novo Nordisk, Biotest, Kedrion, Octapharma and Grifols, acted as a paid consultant for Bayer, Baxter, Pfizer, CSL Behring, Novo Nordisk, Kedrion and Grifols and received unre- stricted research grants from Novo Nordisk and Pfizer. A.I. has received research funds from Bayer, Baxter, Biogen, Novo Nordisk and Pfizer; consultancy fees from Bayer, Pfizer and Novo Nordisk; speaker's honoraria from Bayer, Baxter, Biogen Idec, Biotest, Novo Nordisk and Pfizer; and sits on national and international advisory boards for Biogen Idec, Pfizer and Novo Nordisk. He has received an honorarium to take part in the expert meeting cited in this manuscript and received funds to manage the subsequent Delphi process.

\section{References}

1 Key NS, Negrier C. Coagulation factor concentrates: past, present, and future. Lancet 2007; 370: 439-48.

2 Hoyer LW. Factor VIII inhibitors: a continuing problem. J Lab Clin Med 1993; 121: $385-7$.

3 Wight J, Paisley S. The epidemiology of inhibitors in haemophilia A: a systematic review. Haemophilia 2003; 9: 418-35.

4 Darby SC, Keeling DM, Spooner RJ et al. The incidence of factor VIII and factor IX inhibitors in the hemophilia population of the UK and their effect on subsequent mortality, 1977-99. J Thromb Haemost 2004; 2: $1047-54$.

5 McMillan CW, Shapiro SS, Whitehurst D, Hoyer LW, Rao AV, Lazerson J. The natural history of factor VIII:C inhibitors in patients with hemophilia A: a national cooperative study. II. Observations on the initial development of factor VIII:C inhibitors. Blood 1988; 71: 344-8.

6 Kempton CL, Soucie JM, Abshire TC. Incidence of inhibitors in a cohort of 838 males with hemophilia A previously treated with factor VIII concentrates Journal of thrombosis and haemostasis. Thromb Haemost 2006; 4: 2576-81.

7 Astermark J. Prevention and prediction of inhibitor risk. Haemophilia 2012; 18(Suppl 4): $38-42$.

8 Hay CRM, Palmer B, Chalmers E et al. Incidence of factor VIII inhibitors throughout life in severe hemophilia A in the United Kingdom. Blood 2011; 117: 6367-70.

9 Vermylen J, Peerlinck K. Epidemic of factor VIII inhibitors linked to factor VIII-P. Acta Clin Belg 1991; 46: 419-20.
10 Rosendaal FR, Nieuwenhuis HK, Van den Berg HM et al. A sudden increase in factor VIII inhibitor development in multitransfused hemophilia A patients in The Netherlands. Dutch Hemophilia Study Group. Blood 1993; 81: 2180-6.

11 Giles AR, Rivard GE, Teitel J, Walker I. Surveillance for factor VIII inhibitor development in the Canadian Hemophilia A population following the widespread introduction of recombinant factor VIII replacement therapy. Transfusion Sci 1998; 19: 139-48.

12 Rubinger M, Lillicrap D, Rivard GE et al. A prospective surveillance study of factor VIII inhibitor development in the Canadian haemophilia A population following the switch to a recombinant factor VIII product formulated with sucrose. Haemophilia 2008; 14: 281-6.

13 Baglin T, Beacham E. Is a change of factor VIII product a risk factor for the development of a factor VIII inhibitor? Thromb Haemost 1998; 80: 1036-7.

14 Rea C, Dunkerley A, Sørensen B, Rangarajan S. Pharmacokinetics, coagulation factor consumption and clinical efficacy in patients being switched from full-length FVIII treatment to B-domain-deleted r-FVIII and back to full-length FVIII. Haemophilia 2009; 15: 1237-42.

15 Singleton E, Smith J, Kavanagh M, Nolan B, White B. Low risk of inhibitor formation in haemophilia patients after a change in treatment from Chinese hamster ovary cellproduced to baby hamster kidney cell-produced recombinant factor VIII. Thromb Haemost 2007; 98: 1188-92.

16 Bacon CL, Singleton E, Brady B et al. Low risk of inhibitor formation in haemophilia A patients following en masse switch in treatment to a third generation full length plasma and albumin-free recombinant factor VIII product $\left(\right.$ ADVATE $\left.^{\circledR}\right)$. Haemophilia 2011; 17: 407-11.

17 Gouw SC, Van der Bom JG, Auerswald G, Escuriola EC, Tedgard U, Van den Berg HM. Recombinant versus plasma-derived factor VIII products and the development of inhibitors in previously untreated patients with severe hemophilia A: the CANAL cohort study. Blood 2007; 109: 4693-7.

18 Gouw SC, Van der Bom JG, Ljung R et al. Factor VIII products and inhibitor development in severe hemophilia A. N Engl J Med 2013; 368: 231-9.

19 Christiansen MLS, Balling KW, Persson E et al. Functional characteristics of $\mathrm{N} 8$, a new recombinant FVIII. Haemophilia 2010; 16: 878-87.

20 Stennicke HR, Kjalke M, Karpf DM et al. A novel B-domain O-glycoPEGylated FVIII (N8-GP) demonstrates full efficacy and prolonged effect in hemophilic mice models. Blood Jan 18 2013; 121: 2108-16.

21 Powell JS, Josephson NC, Quon D et al . Safety and prolonged activity of recombinant factor VIII Fc fusion protein in hemophilia A patients. Blood 2012; 119: 3031-7.

22 Makris M, Calizzani G, Fischer K et al. EUHASS: the European Haemophilia Safety Surveillance system. Thromb Res 2011; 127(Suppl): S22-5.

23 Siegmund B, Pollmann H, Richter H, Orlovic $\mathrm{M}$, Gottstein S, Klamroth R. Inhibitor development against FVIII in previously treated patients with haemophilia A. A retrospective data collection. Hämostaseologie 2010; 30: S37-9. 